\title{
Ophthalmological society of the united Kingdom
}

June 9. 1899

(The operative treatment of myopia.)

Eine Discussion über diesen Gegenstand wurde von R. Cross er-öffnet, der nach einem geschichtlichen Ueberblick und der Angabe der Operations-Technik seine Erfahrungen über 48 operierte Fälle mitteilt, die alle ein gutes Resultat gaben. “Wenigstens „,hatte der Vortragende die Operation in keinem Falle zu bereuen-'. Es wurde stets nur ein-seitig operiert und in der Regel nur Fälle über 15 D. M. In einem Falle nur wurde bei 7 D. M., aber von deutlich progressivem Character, die Operation vorgenommen. Deutliche Glaskörpererkrankungen, Neigung zu intraoculären Blvtungen und $\lambda$ Yeichheit des Bulbus galten als Contraindication für die Operation.

Lang berichtet über 30 17/81le; in 1 Falle trat Ablatio ret. ein; aber dasselbe geschah auch auf dem nicht operierten 2. Auge.

Treacher berichtet über 11, Cargill über 6, Frost über 24, Batten über 13 operierte Augen. (The British med. Journ. June 17. 1899. p. 1467.)

1. Beevor und Gunn, A case of obliteration of a branchof the retinal artery following frequent attacks of temporaryamblyopia.

Beevor und Gunn berichten von einem 34jährigen Manne, der seit seiner Rindheit an vorübergehenden, einige Minuten andauernden Anfällen von Amblyopie gelitten hatte, die vorwiegend das rechte Auge betrafen und ohne Ropfschmerzen einhergingen. Vor 9 Monaten blieb nach einem solchen Anfall der Verlust der oberen Gesichtsfeld-hälfte des rechten Auges zurück; er blieb bestehen und fand in einer Atrophie der unteren Papillenhälfte und in einer Obliteration der Zweige der unteren Hälfte der Art. centr. ret. seinen anatomisclien Aus-druck. Gelegentlich trat auch einmal ein vorübergehender Verlust der-unteren Gesichtsfeldhälfte des linken Auges ein.

2. Recovery of sight after partial occlusion of thecentral artery.

Story berichtet iiber 2 Fälle von typischer Embolie der Central-arterie mit Wiederherstellung des Sehvermögens, das im 1. auf Licht-

Sitzungsberichte.

schein, im 2. auf Fingerzählen in 5 Meter gesunken war. Die Be-handlung hatte in Inhalationen von Amylnitrit und der Verabfolgungvon Jodkali bestanden.,; 3. Traumatic division of both optic nerves.

Scott berichtet von einem 23jährigen Aegypter, der nach einem Selbstmordversuch zu ihm gebraclit wurde. Die Kugel war quer durch den Schädel von der rechten zur linken Schläfe durchgedrungen; beide Augäpfel waren vollständig aus den Augenhöhlen herausgeschleudert worden und hingen 19 resp. $15 \mathrm{~mm}$ herab. Nach ihrer Entfernung zeigte sich, dass beide Augenhöhlen von dem Geschoss durchschlagen und die Nervi optici durchgerissen waren.

Sile $\mathrm{x}$.

Ophthalmolog ical society of the united Kingdom. 
July 7. 189 9. (Brit. med. Journ. July 18.189 9.) Changes in the conjunctiva produced by chronic inflammation. Herbert hat fulgeude anatomische Veränderungen der Conjunctiva durch chron. Eützündung eutstehen sehen:

Die Entwicklung von normalen adenoidem Gewebe aus ge-wöhnlichem Bindegewebe.

Bei folliculärer Conjunctivitis und Trachoma unterscheiden sich die neugebildeteu Follikel von den normalen durch den Gehalt von Stroma und Blutgefässen.

Bei der Rückbildung der Follikel wurden die Zellen entweder in die Lymphgefässe abgefühi't oder sie wurden amoeboid oder sie unterlagen der (hyalinen) Degeneration.

Narbeogewebe kann eutstehen bei der ganz chronisch ver-laufendeii Conjunct, simplex.

Aus den· Trachom-Follikeln können breite Papillen ähnlich wie bei Frühjalirskatarrh eutstehen. Conjunctivalcysten in grosser Zahl können nach chron. und besonders trachomatöser Conj. in der oberen Tarsalmembran sich entwickelu.

Stellwag's ,.brawny trachoma"، scheint auf einer basophilen Degeneration von

Bindegewebsfasern zu beruhen.

Plastic operation for Contracted.

Richardson beschreibt eine neue plastische Operations-Methode zur Beseitigung des geschrumpfted Conjuntival-Sackes.

A case of penetrating wound of the orbit followed by Meningitis. Trephining. Recovery.

R. Rockliffe und Ilainworth berichten über einen Patienten

der 7 Tage. nachdem er eine †iefe Orbitalwunde (durch den Schlag mit einer kupferbeschlagenen Pfeife) bekommen hatte an Meningitis er-krankte. Die anfänglich gestellte Diagnose auf einen in der 1. Temporal-Region lokalisierten Abscess erwies sich bei der Trepanation als falsch ; dagegen wurde aus dem Seitenventrikel eine beträchtliche Menge Flüssigkeit entleert. Nach 4 Wochen trat líeilung ein. 